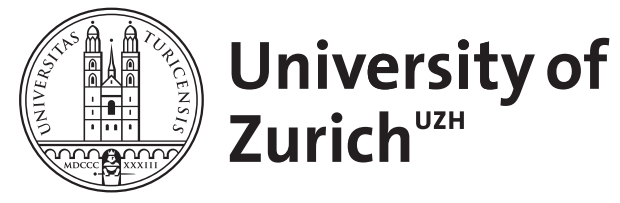

Zurich Open Repository and Archive

University of Zurich

University Library

Strickhofstrasse 39

CH-8057 Zurich

www.zora.uzh.ch

Year: 2011

Der Patient im Mittelpunkt - das Geld im Vordergrund

Rössler, W ; Folkers, G

DOI: https://doi.org/10.1024/1661-8157/a000714

Posted at the Zurich Open Repository and Archive, University of Zurich

ZORA URL: https://doi.org/10.5167/uzh-58369

Journal Article

Published Version

Originally published at:

Rössler, W; Folkers, G (2011). Der Patient im Mittelpunkt - das Geld im Vordergrund. Schweizerische Ärztezeitung, 92(48):1881-1883.

DOI: https://doi.org/10.1024/1661-8157/a000714 


\section{Der Patient im Mittelpunkt - das Geld im Vordergrund}

Wulf Rössler ${ }^{a, b}$,

Gerd Folkers ${ }^{b}$

a Psychiatrische Universitätsklinik Zürich

b Collegium Helveticum in gemeinsamer Trägerschaft von Universität Zürich und ETH Zürich

Interessenverbindungen:

Wulf Rössler

Verbindungen zur Pharmazeutischen Industrie

Eli Lilly (Advisory Board: Olanzapine)

Janssen-Cilag AG (Advisory Board: Paliperidone Palmitate)

AstraZeneca AG (Advisory Board: Quetiapin)

Teilnahme an diversen

Symposien mit eigenständigen, nicht-pharmazeutischen

Vorträgen, die zum Teil von der Pharmazeutischen Industrie gesponsert wurden

Gerd Folkers

Verbindung zur

Pharmazeutischen Industrie

AZAD Pharma Schaffhausen:

Board of Directors
Das Schweizer Gesundheitswesen findet international viel Anerkennung. Dies hat aber seinen Preis: Die Schweiz nimmt weltweit den dritten Platz bei den höchsten Gesundheitsausgaben ein [1].

Seit vielen Jahren versucht die Gesundheitspolitik, die Kosten der Gesundheitsversorgung zu senken. Die Erfolge dieser Bemühungen sind jedoch bisher eher bescheiden; allenfalls liess sich punktuell der Kostenanstieg bremsen. Die Gründe, die für die ständig weitersteigenden Kosten genannt werden, sind z. B. der demografische Wandel oder der medizintechnologische Fortschritt.

Zumindest in der medialen Aufmerksamkeit kommt der Senkung der Medikamentenkosten hohe Priorität zu. Es besteht ein besonderes Misstrauen gegenüber der pharmazeutischen Industrie, deren zentraler Erfolgsparameter der finanzielle Ertrag aus ihrer wirtschaftlichen Tätigkeit ist.

Ein Ansatz zur Kostensenkung liegt in der Verschreibung (kostengünstigerer) Generika anstelle von Originalpräparaten. Gemäss dem Schweizerischen Heilmittelinstitut Swissmedic [2] sind Generika «Arzneimittel, welche sich als Nachahmer an ein beim Institut zugelassenes Originalpräparat anlehnen. Sie zeichnen sich durch gleichen Wirkstoff (...), gleiche Darreichungsform, gleichen Applikationsweg und gleiche Indikationen aus. Sie sind mit dem Originalpräparat austauschbar.» Dabei spielt die sogenannte «therapeutische Äquivalenz» eine zentrale Rolle: «Unter therapeutischer Äquivalenz versteht man innerhalb gewisser Grenzen (üblicherweise $+/-20 \%$, je nach therapeutischer Breite und Risiko von Neben-

\section{Le patient au centre des préoccu-} pations - I'argent aux avant-postes

La prise en charge médicale en Suisse est d'un excellent niveau mais elle a aussi un coût. Depuis des années, la politique de la santé tente de limiter la hausse des coûts du système de santé. Une des mesures pour réduire les frais consiste à prescrire des génériques. Cependant, cette pratique peut présenter des inconvénients considérables pour les patients.

dingungen können auch die Darreichungsformen abweichen

\section{Anreize zur Kostensenkung}

Vor diesem Hintergrund können Generika in der Regel billiger angeboten werden als Originalpräparate. Die pharmazeutische Industrie sieht sich ihrerseits gezwungen, die Preise nach Ablauf des Patentschutzes ihres Präparates zu senken. So können gesamtwirtschaftlich beträchtliche Einsparungen erzielt werden.

Um die Akteure im Gesundheitswesen zur Umstellung von Originalpräparaten auf ein Generikum zu motivieren, werden finanzielle Anreize geschaffen. So kann sich für den Patienten je nach Medikament der Selbstbehalt ändern. Die Apotheker, die ein Generikum anstelle eines Originalpräparates verkaufen,

\section{«Generika bedürfen lediglich des Nachweises der Bioäquivalenz mit einer Abweichung von +/-20\%.»}

Korrespondenz:

Prof. Dr. med. Dipl.-Psych. Wulf Rössler

Klinikdirektor Psychiatrische Universitätsklinik Zürich Klinik für Soziale Psychiatrie und Allgemeinpsychiatrie ZH West

Militärstrasse 8

Postfach 1930

CH-8021 Zürich

roessler[at]dgsp.uzh.ch

www.puk-west.uzh.ch wirkungen, jedoch auch weiter und enger) identisches Wirksamkeits- und Nebenwirkungsprofil zweier Arzneimittel (...) Nachdem der Nachweis der therapeutischen Äquivalenz einen sehr grossen, häufig unzumutbaren Aufwand in Form von klinischen Studien darstellt, wird er in der Regel indirekt durch den Nachweis der Bioäquivalenz geführt.»

Dies bedeutet in der Markteinführung von Generika, dass für Generika kein erneuter Wirksamkeitsnachweis benötigt wird. Generika bedürfen lediglich des Nachweises der Bioäquivalenz mit einer Abweichung von +/-20\%. Unter gewissen Be- erhalten eine Beratungspauschale. Ärzte ihrerseits stehen unter einem negativen finanziellen Anreiz über den Kostenindex.

Während andere Länder erfolgreich die Verschreibung von Generika gefördert haben [3], ist der Anteil der Generikaverschreibungen in der Schweiz mit $14 \%$ in 2010 allerdings noch gering [4].

\section{Profitiert der Patient von der Einführung eines Generikums?}

Für den Patienten selbst ist der persönliche Nutzen der Preissenkung bzw. der niedrigere Preis des Gene- 


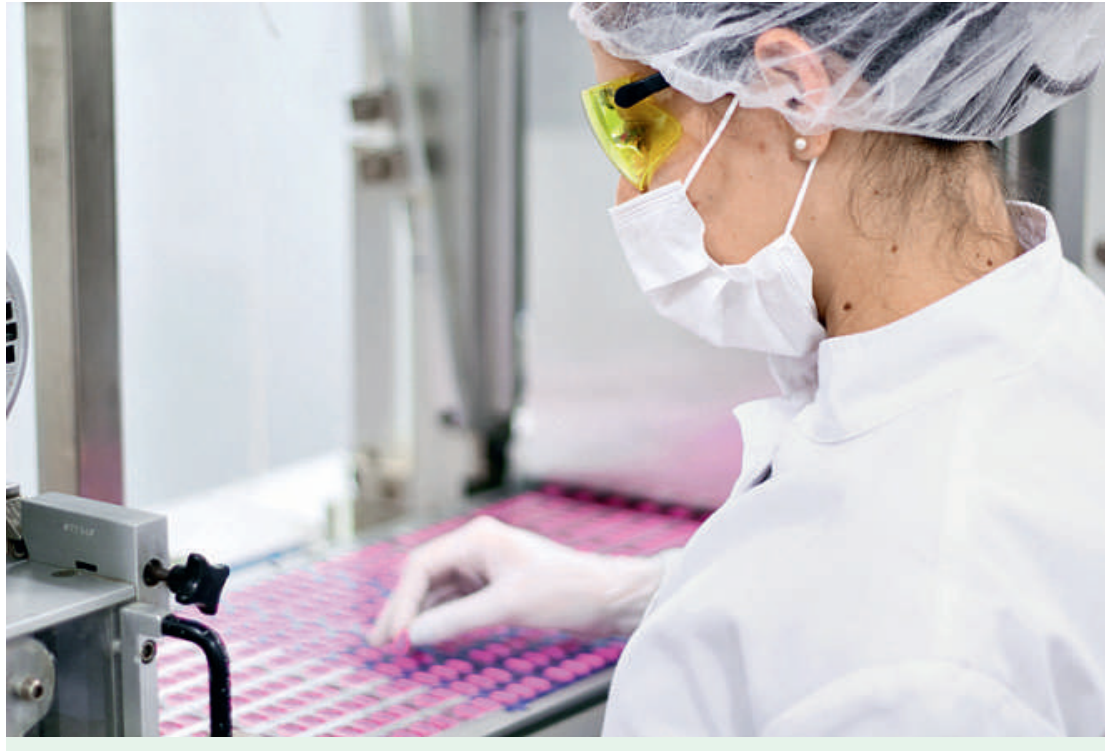

Der Medikamentenherstellung geht bei Originalpräparaten ein langer Forschungs- und Entwicklungsprozess voraus. Er entfällt bei Generika.

rikums zunächst gering, weil - insbesondere bei chronischen Erkrankungen - seine Franchise unabhängig vom Preis des Präparates schnell aufgebraucht sein wird. Die Solidargemeinschaft der Versicherten, die dem Patienten unabhängig von seiner finanziellen Leistungsfähigkeit im Krankheitsfall immer eine wirksame Behandlung garantiert, appelliert an den Patienten, seiner Verpflichtung des sparsamen Umgangs mit finanziellen Ressourcen nachzukommen. Kann sich aber der Patient sicher sein, dass ihm daraus keine Nachteile erwachsen?

Wie erwähnt, erlaubt die therapeutische Äquivalenz zwischen einem Originalpräparat und einem Generikum eine Varianz von $+/-20 \%$. Dies bedeutet, dass ein Patient bei zwei Verordnungen eines Originalpräparats zwei unterschiedliche Generika mit einer Spannbreite von 40\% erhalten kann.

Die Bioverfügbarkeit wird ermittelt als «Area under the Curve» (AUC), die die Fläche unter einer Verteilung beschreibt. Parameter sind die Resorptionsgeschwindigkeit und die resorbierte Menge. Impliziert werden gleiche Metabolisierungsraten, weil der Inhaltsstoff in Originalpräparat und Generikum identisch ist. Abweichend kann die Freisetzung des Wirkstoffs aus der Arzneiform sein, die im Wesentlichen von der galenischen Formulierung bestimmt wird. Der Patient kann also bei einer Umstellung beträchtlichen Schwankungen in der Wirkstoffkonzentration unterworfen sein. «Zu viel» oder «zu wenig» eines Medikamentes kann die klinische Wirksamkeit erheblich beeinträchtigen.

Hinzu kommt, dass von einem Generikum nicht zwangsläufig alle Darreichungsformen vorhanden sein müssen. Der Patient ist dann unter Umständen gezwungen, Tabletten zu teilen, was die ursprünglich verschriebene Dosierung zu einem Glücksspiel macht, vor allem, wenn der Patient unter kognitiven und/oder sensorischen Beeinträchtigungen leiden sollte.

Ein weitgehend unterschätzter Effekt ist auch die psychologische Haltung eines Patienten gegenüber einem neuen oder anderen Medikament. Die Umstellung einer Dauermedikation eines Originalpräparates auf ein Generikum kann in einer nicht geringen Anzahl von Fällen zu Unverträglichkeiten führen. Dies kommt sogar dann vor, wenn das Generikum nicht nur im Wirkstoff identisch ist, sondern auch in Begleitstoffen und Darreichungsform. Nur die Verpackung und der Name unterscheiden sich vom Original. Das wird landläufig als Placebo-Effekt bezeichnet [5].

Zuletzt soll erwähnt werden, dass Ärzte in der Regel die von ihnen verordneten Medikamente in ihrem Wirkungs- wie Nebenwirkungsprofil bestens kennen und vor diesem Hintergrund ihre Patienten über Wirkungseintritt, Art der Wirkung wie der Nebenwirkungen mit der notwendigen Sicherheit fundiert beraten können. Diese Sicherheit geht verloren, wenn der Patient ein dem Arzt in der Wirksubstanz zwar bekanntes, aber in vielen anderen Parametern unbekanntes Medikament erhält.

\section{Konsequenzen für die Pharmazeutische Industrie?}

In der Diskussion um Generika geht häufig ein wesentlicher Aspekt vergessen: Nur weil es Patentschutz für Originalpräparate gibt, können billigere Nachahmerprodukte nach Ablauf der Patentzeit entwickelt werden. Denn ein Patent bedingt die vollständige Offenlegung. Gäbe es diese nicht - wie bei Coca Cola, das nie patentiert wurde - stünden heute einige der breit verwendeten Arzneimittel nicht als kostengünstige Variante zur Verfügung. Ein ausreichender Patentschutz und als dessen Konsequenz ein teures Originalpräparat dient also der Pharmazeutischen Industrie und den Patienten. Erstere kann lohnend in komplexe Arzneimittelentwicklung investieren, letztere erhalten ein nach neuesten Erkenntnissen hergestelltes Therapeutikum, das nach 20 Jahren frei verfügbar für ein Nachahmer-Präparat wird. Dieses System wird immer stärker in Frage gestellt und auch durch juristische Strategien, wie «Ergänzende Schutzzertifikate» aufseiten der Erfinder und «Versuchsprivilegien» für die Generikahersteller unterlaufen.

Als Konsequenz beobachtet man bei der pharmazeutischen Grossindustrie eine zunehmende Verlagerung der Entwicklungsrisiken auf Partnerschaften mit externen Entwicklungsfirmen oder die Risikoverlagerung auf akademisch-klinische Zentren.

Andererseits ist eine Tendenz zur Abkehr von der Entwicklung breit anwendbarer Arzneimittel und eine Hinwendung zu hochpreisigen Spezialsegmenten im Bereich von Tumor-, neurodegenerativen und/ oder seltenen, genetisch bedingten Erkrankungen festzustellen. Neben ökonomischen Überlegungen zeigt sich hier die Auswirkung einer molekular definierten Spitzenmedizin. Je präziser der Blick auf die Kausalität einer Erkrankung und das daraus entwi- 
ckelte Medikament, umso mehr schrumpft die Population an Patienten, die exakt der gleichen Therapie bedürfen. Am Ende der Entwicklung steht das «massgeschneiderte Medikament», das für einen einzelnen Menschen entwickelte Arzneimittel.

Gleichermassen kompliziert ist die Lage bei den Biosimilars. Hier handelt es sich um Nachahmerprodukte von proteinbasierten Arzneimitteln, wie beispielsweise kanzerostatisch wirksame Antikörper, die zu den teuersten Medikamenten überhaupt zählen. Im Gegensatz zu herkömmlichen synthetischen Arzneistoffen enthalten Biosimilars eine Reihe von biotechnologischen Produktionsprozessen. Je nach verwendetem tung für jede in der Gesundheitsversorgung tätige Person. Allerdings darf Sparsamkeit nicht zu schwer kalkulierbaren Risiken für die Patienten führen. Die Idee, wo immer möglich Originalpräparate durch Generika (oder Generika durch noch billigere Generika) $\mathrm{zu}$ ersetzen, wird weder den Patienten noch den gegenwärtigen Trends in der Medikamentenentwicklung gerecht.

Es scheint vernünftig, auch bezüglich der behördlichen Ansprüche an Arzneimittel, einen anderen Weg einzuschlagen als den der maximalen Sicherheit bei maximaler Wirkung, ein Denken, das sich aus der Contergan-Affäre ableitet.

\section{«Der Patient kann also bei einer Umstellung beträchtlichen Schwankungen in der Wirkstoffkonzentration unterworfen sein.»}

Stamm eines möglicherweise global vorkommenden Mikroorganismus und je nach Standardisierungsprozess können sognannte post-translationale Modifikationen die «Zuckerhülle» der Eiweisse so verändern, dass es zu erheblichen pharmakokinetischen Parameteränderungen oder zu unerwünschten Immunreaktionen kommt. Eine ökonomisch begründete einfachere Zulassungspraxis scheint bei den hochkomplexen Wirkungsweisen dieser Arzneimittel fraglich.

Mit der Strategie der «Medikamenten-Repositionierung» hat die Pharmaindustrie in der letzten Dekade einen neuen Weg eingeschlagen, um nicht mehr ertragsreiche Medikamentenlinien zu stärken. Verkürzt geht es darum, die bekannten und im Interesse der Hauptwirkung tolerierten Nebenwirkungen in eine neue therapeutische Anwendungsmöglichkeit umzumünzen. Zweifellos ist dies auch einem erhöhten Kostendruck durch Generika geschuldet. So wurden in den letzten Jahren beispielsweise eine Reihe von Antidepressiva, meist vom Typ SSRI, neu profiliert. In den klinischen Studien sind diese SSRIs nun als Mittel zur Raucherentwöhnung, zur Behandlung von Fibromyalgie, der prämenstruellen Dysphorie oder von stressbedingter Harninkontinenz in Entwicklung [6]. Neue Indikationen sind oft in bisher klinisch «vernachlässigten» Gebieten zu finden, bedingen deswegen die Formulierung neuer Endpunkte in den Studien für denselben Wirkstoff. Dann aber können solche Repositionierungen wie z.B. bei Aspirin ${ }^{\circledR}$ äusserst erfolgreich sein. Aspirin ${ }^{\circledR}$ erlebt als kardioprotektives Therapeutikum eine Renaissance als Originalpräparat, von dem es inzwischen wiederum eine Reihe von Generika gibt.

\section{Auf dem Weg zu einem rationaleren Gesundheitswesen}

Der sparsame Umgang mit finanziellen Ressourcen des Gesundheitswesens ist eine ethische Verpflich-
Das Beispiel der «Medikamenten-Repositionierung» zeigt, dass pharmakokinetische Optimierung, abweichende Dosierungsschemata, geänderte Applikationsformen und neue molekulare Mechanismen wesentliche Teile der Repositionierungsstrategie sind. Hier sollte die Rolle der Generika und «Me-too-Präparate» überdacht werden. Bestünden genügend ökonomische Anreize und weniger administrative Barrieren, sich um die weitere Erforschung und Profilierung existierender Wirkstoffe in neuen Anwendungsgebieten zu kümmern, könnte das von grösserem medizinischem und gesellschaftlichem Nutzen sein als ein breites Angebot möglichst billiger Nachahmerprodukte. Und zuletzt werden neue gesundheitspolitische Strategien erforderlich, um den neueren Entwicklungen wie Biosimilars und massgeschneiderten Medikamenten für den einzelnen Patienten gerecht zu werden.

\section{Literatur}

1 Gesundheit. Panorama. Bundesamt für Statistik. 2011; Februar.

2 Anleitung des Schweizerischen Heilmittelinstituts für Humanarzneimittel mit bekannten Wirkstoffen. 3. Dezember 2002.

3 Richens A. Impact of Generic Substitution of Anticonvulsants on the Treatment of Epilepsy. CNS Drugs. 1997;8(2):124-33.

4 Dank Generika könnten Gesundheitskosten zusätzlich um 650 Millionen Franken entlastet werden. Generika News. Intergenerika. 2010; Februar/März.

5 Krummenacher P, Candia V, Folkers G, Schedlowski M, Schönbächler G. Prefrontal cortex modulates placebo analgesia. Pain. 2010;148:368-74.

6 Ashburn TT, Thor KB. Drug repositioning: identifying and developing new uses for existing drugs. Nat Rev Drug Discov. 2004;3(8):673-83. 